\title{
Anisotropy effect of magnetoresistance in spin polarized tunneling
}

\author{
Ching-Ray Chang and Sui-Pin Chen \\ Department of Physics, National Taiwan University, Taipei, Taiwan, ROC,
}

\begin{abstract}
We calculate the influence of single-ion anisotropy in spin polarized tunneling and conclude that the tunneling magnetoresistance ratio increases for lower barrier height with the increasing anisotropy parameter. In particular, the optimal alignment of the ferromagnetic layers, yielding the largest TMR ratio is proposed.
\end{abstract}

\section{INTRODUCTION}

Large magnetoresistance(MR) observed in ferromagnetinsulator-ferromagnet (FM//FM) tunneling junctions [1-2] has attracted considerable attention for its potential applications in sensors and magnetic random access memory[3]. The resistance of a FM/L/FM tunneling junction is usually much higher than that of ferromagnetic multilayers. However, its magnetoresistance, ratio is still comparable to the giant magnetoresistance (GMR) of multilayers[4]. For high resistance tunneling junctions, the power consumption is much less than in multilayers, which attract attention not only for fundamental studies but also for application of the junction as an electronic device. If two ferromagnetic electrodes are separated by a nonmagnetic tunneling barrier, then the spin polarized tunneling conductance depends on a number of parameters, e.g., relative angle between the magnetizations of two ferromagnetic electrodes, the applied field, the bias voltage and the temperature. The observed magnetoresistance is attributed to the polarized electron tunneling across the insulating barrier for the negligible contribution of ferromagnetic electrodes, and it is called tunneling magnetoresistance (TMR).

An early work reported[5] that the tunneling conductance of an $\mathrm{Fe} / \mathrm{Ge} / \mathrm{Co}$ junction has an MR ratio up to $14 \%$ at $4.2 \mathrm{~K}$ under zero bias voltage. Julliere qualitatively analyzed TMR ratio, $\Delta G / G$, using Tedrow and Meservey's theory [6] and found that it could be expressed as

$$
\frac{\Delta G}{G}=\frac{G_{p}-G_{a p}}{G_{p}}=\frac{2 P_{1} p_{3}}{\left(1+P_{1} P_{3}\right)},
$$

where $G_{p}$ and $G_{a p}$ stand. for the conductance of two ferromagnetic electrodes with parallel and antiparallel magnetizations, respectively. $P_{7}$ (or $P_{3}$ ) are the polarizations

Manuscript teceived February 25, 1999.

Ching-Ray Chang, 886-2-23626937 ext. 324, fax 886-2-2363-9984, crchang@phys.ntu.edu.tw. S. P.Chen

This work was supported in part by Taiwan Economics Minister under Grant No. 88S12-J3. of the two ferromagnets. The upper bound of the experimental data for TMR ratio is roughly proportional to the product of the spin polarizations of both ferromagnets, but the spread of those data is very broad[7]. Based on the free electron model, Slonczewski suggested that TMR ratio depends on the spin polarization of conduction electrons [8]. However, spin polarization decreases fast with the increase of the barrier height in Slonczewski's theory and the predicted value of the TMR ratio is much smaller than experimental values[2]

Here we study the effect of single-ion anisotropy $[9,10]$ of tunneling electrons on the tunneling junction. The spin and charge transmission coefficients are calculated from a proposed spin Hamiltonain and the TMR then follows. $\mathrm{Fe} / \mathrm{l} / \mathrm{Fe}$ junction is chosen to demonstrate the anisotropy effect of the ferromagnetic electrodes and the optimal alignment of the ferromagnetic layers, yielding the largest TMR ratio is proposed.

\section{THEOTY}

Our analysis is based on the plane wave approximation of the spin-polarized conduction electrons within the three junction regions: two ferromagnetic electrodes and a rectangular barrier(Fig. 1). The transmission of a single electron transversing a rectangular barrier from the left into the right ferromagnetic electrode can be characterized by the longitudinal part of the spin Hamiltonian

$$
H_{\xi, i}=-\frac{\hbar^{2}}{2 \mu}\left(\frac{d}{d \xi}\right)_{i}^{2}+U_{i}(\xi)-h_{j}(\xi) \sigma_{i}+D_{i}\left(\sin \theta_{i}\right)^{2} \text {. }
$$

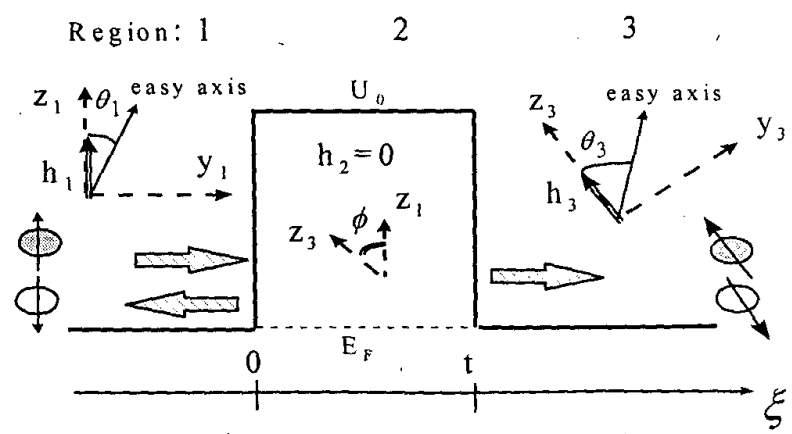

Figure 1: Schematic diagram for a FM//FM tunneling junction. Easy axis of each ferromagnetic layer deviates $\theta_{j}$ from their spin quantization axis. The molecular field $h_{i}$ is along the spin quantization axis $z_{i}$ and the angle between $z_{1}$ and $z_{3}$ is $\phi$. 
Subscripts $i(=1,2,3)$ represent the ferromagnetic layer $F_{I}$, the barrier, and the right ferromagnetic layer $F_{3}$, respectively. The kinetic energy term is $-\hbar^{2} / 2 \mu(\mathrm{d} / \mathrm{d} \xi)^{2}, U$ is the potential term, $\mu$ is the effective mass and $\hbar$ is the Planck constant. The internal exchange energy of the $i$-th ferromagnetic layer is $-h_{i}(\xi) \sigma_{i}$, where $h$ is the molecular field and $\sigma$ is the Pauli spin operator. We also included the single-ion anisotropic term $[9,10], D_{i}\left(\sin \theta_{i}\right)^{2}$, where $D_{i}$ is the anisotropy parameter $[9,10]$ and $\theta_{i}$ is the angle between the spin quantization axis and the easy axis in the $i$-th layer. Since the ferromagnetic materials in electrodes can be different, $h_{l}$ does not necessary equal $h_{3}$. The spin quantization axes $z_{1}$ and $z_{3}$ span an angle $\phi$ and we assume the directions of $h_{1}$ and $h_{3}$ to be parallel with the corresponding spin quantization axes $z_{1}$ and $z_{3}$ (Fig. 1).

Consider an incident plane wave of a spin-up electron with unit flux in the $F_{l}$. layer $\xi<0$ as shown in Fig. 1): by matching boundary conditions at the interfaces $\xi=0$ and $\xi=$ $t$, we can determine the wave function in three regions. In our calculations, we assume a rectangular barrier for $0<\xi<t$, and $h_{2}=0$ inside the barrier(Fig. 1). After some algebraic manipulations and spinor transformation, the transmission probability $\left(\mathrm{T}_{\sigma \sigma}\right)$ of tunneling electrons from the spin- $\sigma$ state in the $F_{l}$ layer to the spin- $\sigma$ ? state in the $F_{3}$ layer become

$$
\begin{aligned}
& T_{\uparrow \uparrow}=8 \frac{k_{\uparrow} k_{\uparrow} \cdot \kappa^{2} e^{-2 \kappa t}}{\left(\kappa^{2}+k_{\uparrow}{ }^{2}\right)\left(\kappa^{2}+k_{\uparrow^{2}}{ }^{2}\right)}(1+\cos \phi), \\
& T_{\uparrow \downarrow}=8 \frac{k_{\uparrow} k_{\downarrow} \cdot \kappa^{2} e^{-2 \kappa t}}{\left(\kappa^{2}+k_{\uparrow}{ }^{2}\right)\left(\kappa^{2}+k_{\downarrow}{ }^{2}\right)}(1-\cos \phi) .
\end{aligned}
$$

Since $\kappa \gg 1$, and thus for brevity, we only keep the terms up to $\mathrm{e}^{-2 \mathrm{kt}}$. The wave vector inside the ferromagnetic layers is $k_{\sigma}$ and the imaginary wave vector inside the insulating barrier is $i \kappa$. The values of $T_{\downarrow \downarrow}(\phi)$ and $T_{\downarrow \uparrow}(\phi)$ follow by symmetry. Since the anisotropy term in the spin Hamiltonian only depends on the angle $\theta$, the contribution to the potential energy by either spin-up or spin-down electrons is the same. Therefore, even if considering the single-ion anisotropy, the energy splitting of the spin-up and spin-down electrons remains the same as they are isotropic. However, the kinetic term (i.e., wave vector, $k_{\sigma}$ ) becomes a function of $D$ and $\theta$ for the changing of potential energy from single-ion anisotropy effect. In order to simplify the resultant expression for tunneling conductance, we introduce the following notation:

$$
\begin{aligned}
n_{1}\left(\theta_{1}, d_{1} ; p_{1}\right) & =\frac{k_{\downarrow}\left(\theta_{1}, d_{1} ; p_{1}\right)}{k_{\uparrow}\left(\theta_{1}, d_{1} ; p_{1}\right)} \\
& =\sqrt{\frac{\left(1+p_{1}^{2}\right)-\left(1-p_{1}^{2}\right)\left(1+d_{1} \sin ^{2} \theta_{1}\right)}{\left(1+p_{1}^{2}\right)+\left(1-p_{1}^{2}\right)\left(1-d_{1} \sin ^{2} \theta_{1}\right)}}, \\
g_{1}\left(\theta_{1}, d_{1} ; p_{1}, m_{1}\right) & =\frac{\kappa}{k_{\uparrow}\left(\theta_{1}, d_{1} ; p_{1}\right)} \\
& =m_{1} \sqrt{\frac{2}{\left(1+p_{1}^{2}\right)+\left(1-p_{1}^{2}\right)\left(1-d_{1} \sin ^{2} \theta_{1}\right)}}
\end{aligned}
$$

where $p_{1}\left(=k_{\downarrow} / k_{\uparrow}\right)$ and $m_{1}\left(=k_{k} / k_{\uparrow}\right)$ are isotropy ratios, i.e., $D_{1}=0$. We let $d_{1}=D_{1} / h_{1}\left|\sigma_{l z}\right|$, and $d_{3}=D_{3} / h_{3}\left|\sigma_{3 z}\right|$. If $D_{1}=D_{3}$, we have

$$
d_{3}=\frac{h_{1}}{h_{3}} d_{1}=\frac{\left(1-p_{1}^{2}\right)\left(1+p_{3}^{2}\right)}{\left(1+p_{1}^{2}\right)\left(1-p_{3}^{2}\right)} d_{1}
$$

Similar expressions for $n_{3}$ and $q_{3}$ can also be given. Using the usual techniques[8], we sum the charge transmission over $E_{\xi}$ and $k_{/ /}$for all occupied states near the Fermi energy $\left(E_{\mathrm{F}}\right)$ at $0 \mathrm{~K}$, and obtain the tunneling conductance at vanishing external voltage[8,11]. In the following calculation of tunneling conductance, $\kappa$ and $k_{\sigma}$ therefore are only derived from $\mathrm{E}_{\mathrm{F}}$. For a $F_{1} / I / F_{3}$ junction, the tunneling conductance depends on the angle $\phi$ between the two magnetizations, and we can write it as

$$
\begin{aligned}
& G(\phi)=\frac{e^{2} \kappa}{\pi^{2} \hbar t} e^{-2 \kappa t} \frac{\left(q_{1}^{2}+n_{1}\right)\left(1+n_{1}\right) q_{1}}{\left(q_{1}^{2}+1\right)\left(q_{1}^{2}+n_{1}^{2}\right)\left(q_{3}^{2}+n_{3}\right)\left(1+n_{3}\right) q_{3}} \\
& {\left[1+\frac{\left(1-n_{1}\right)\left(q_{1}^{2}-n_{1}\right)}{\left(1+n_{1}\right)\left(q_{1}^{2}+n_{1}\right)} \frac{\left(1-n_{3}\right)\left(q_{3}^{2}-n_{3}\right)}{\left(1+n_{3}\right)\left(q_{3}^{2}+n_{3}\right)} \cos \phi\right] \text {, }}
\end{aligned}
$$

The angular dependence of the tunneling conductance $G(\phi)$ has predominantly cosine dependence[8] apart from modifications due to $k_{\sigma}$.

\section{RESULTS AND CONCLUSIONS}

We explore the tunneling conductance and the TMR ratio under different orientations of the ferromagnetic easy axes and of the applied field. For an $\mathrm{Fe} / \mathrm{l} / \mathrm{Fe}$ junction, we study three geometric arrangements: (a)Perpendicular : The easy axes of both ferromagnetic layers are perpendicular to the $z$ axis (Fig.2(a)). (b)Oblique: Both easy axes deviate from the applied field, e.g., the angle of easy axes are $\pm 60^{\circ}$ with the $z$ axis (Fig.2(b)). (c)Orthogonal: The easy axes of two ferromagnetic layers are orthogonal to each other, and one of the axes is along the z-axis (Fig.2(c)). For an applied field along the $z$-axis, TMR ratio of the trilayer thin film shows a strong dependence on the easy axes orientation of two ferromagnetic layers. A large enhancement of the TMR value may be achieved by the proper alignment of the two ferromagnetic layers. Our calculations suggest that the perpendicular configuration always gives the largest TMR ratio for a lower barrier height. When applied field is perpendicular to the anisotropy axis, then the $k_{\sigma}$ value for the demagnetized and saturated states significantly differ and huge TMR ratio can be observed. At lower barrier heights, TMR ratio is sensitive to the anisotropy parameter(Fig. 2), whose influence becomes insignificant at higher barrier heights. When the barrier heights increase, and then $\mathrm{D} / \mathrm{U}_{0}$ gradually decreases; therefore, the influence of single-ion anisotropy become insignificant as $\mathrm{D} / \mathrm{U}_{0}$ approaching zero. In other word, the orientations of two ferromagnetic electrodes will not affect the TMR ratio at higher barrier heights(Fig. 2). 
(a)

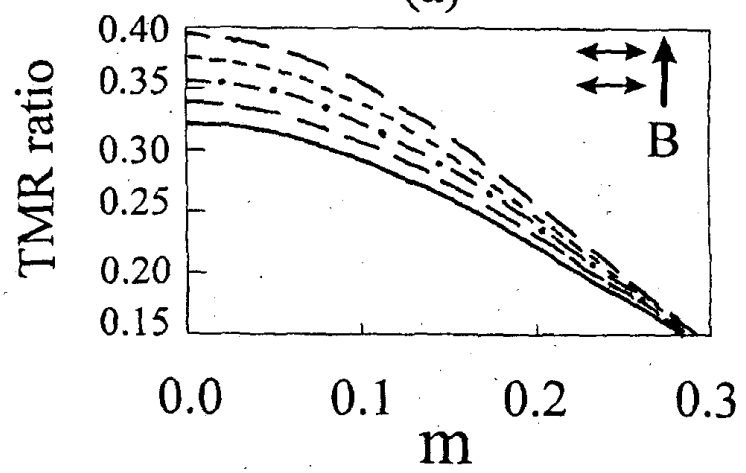

(b)

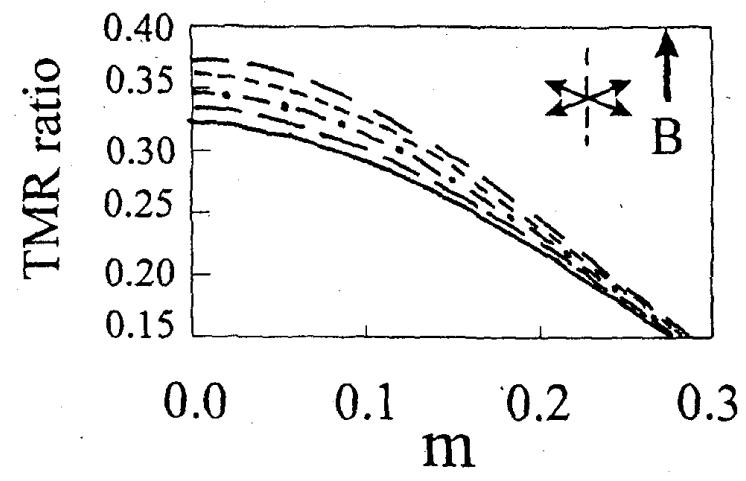

(c)

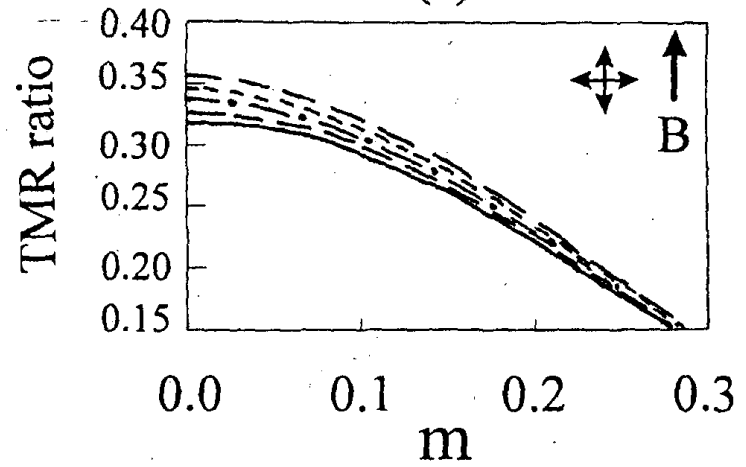

Figure 2: TMR ratio versus barrier height of $\mathrm{Fe} / \mathrm{I} / \mathrm{Fe}$ junctions with three arrangements of their easy axes.(a) Perpendicular: The easy axes of both ferromagnetic layers are all perpendicular to the $z$ axis. (b)Oblique: Both easy axes deviate from the applied field, e.g., the angle of easy axes are $\pm 60^{\circ}$ with the $z$ axis. (c)Orthogonal: The easy axes of two ferromagnetic layers are orthogonal to each other, and one of the easy axis is along the $z$-axis. The wave vectors of $\mathrm{Fe}$ in calculation are $k_{\uparrow}=1.09 \AA^{-1}$, and $k_{\downarrow}=0.43 \AA^{-1}[8]$.

Anisotropy constant $d=D / h\left|\sigma_{z}\right|$ and barrier heights $m$ are used to be parameters. The graphs on the figures are $d_{1}=0.0$ (solid line), $d_{1}=0.025$ (longdash line), $d_{1}=0.05$ (dash-dotted line) and $d_{1}=0: 075$ (short-dash line) and $d_{1}=0.1$ (long-dash line), respectively
If the easy axes of the ferromagnetic layers both parallel to the applied field, it is the same results as isotropy (i.e., $D=0$ in Fig. 2) for $\theta=0$ now. Arbitrary anisotropy constants used in Fig. 2 are between the reported bulk values of transition metals and rare earth, in other words, TMR ratio is probably a little overestimated for the transition metal. However, the anisotropy constant of thin film is larger than its bulk value, and the anisotropy values in Fig. 2 are still much smaller than the values frequently used in theoretical modeling[10,11]. In particular, anisotropy constant of FePt is known to larger than the pure transition metal, and is about the same order used in Fig. 2. Therefore, the single-ion effect should be observable with the proper materials and orientation of easy axes. Indeed, our results qualitatively agree with the experimental data for $\mathrm{NiFe} / \mathrm{MgO} / \mathrm{Co}$ junctions[12]. Our calculation also indicated that the possible origins of the scattered data for TMR may be due to a distribution of the orientation of the easy axes and due to the variation of barrier heights. A further experimental study is required to clarify the contribution of the single-ion anisotropy in the TMR junction.

In summary, we have calculated that the influence of single-ion anisotropy on the tunneling magnetoresistance with different barrier heights. It shows that TMR ratio can be enhanced with the proper orientation of the easy axes of two ferromagnetic electrodes at lower barrier heights. We conclude that the TMR ratio in the perpendicular situation should be the largest among all possible arrangements.

\section{REFERENCE}

[1]T. Miyazaki, T. Yaoi and S. Ishio, "Large magnetoresistance effect in $82 \mathrm{Ni}-\mathrm{Fe} / \mathrm{Al}-\mathrm{Al}_{2} \mathrm{O}_{3} / \mathrm{Co}$ magnetic tunneling junction", J. Magn. Magn Mater, vol. 98, L7-8, Dec. 1991:

[2]J. S. Moodera, Lisa R. Kinder, Terrilyn $M$. Wong and $R$ Meservey,"Large magnetoresistance at room temperature in ferromagnetic thin film tunneling junction", Phys. Rev. Lett., vol. 74, pp. 3273-3276, 1995. J. S. Moodera and Lisa R. Kinder, J. Appl. Phys., 79, 4724,1996

[3]J. L. Simonds, "Magnetoelectronics: Today and Tomorrow", Physics Today, pp. 26-30, Apr., 1995.

[4]P. M. Levy, in Solid State Physics, edited by H. Ehrenreich and D. Turnbull (Academic, New Yòrk, 1994) vol. 48, pp. 367-463

[5]M. Julliere, "Tunneling between ferromagnetic films", Phys. Lett. A, vol. 54 , pp. 225-226, 1975 .

[6]P. M. Tedrow and R. Meservery, "Spin-dependent tunneling into ferromagnetic Nickel", Phys. Rev. Lett., vol. 26, pp. 192-195, 1971.

[7] T. Miyazaki and N. Tezuka, "Spin polarized tunneling in ferromagnet/insulator/ferromagnet junctions, J. Magn. Magn. Mater., vol. 151, pp. 403-410, Dec. 1995

[8]J. C. Slonczewski, "Conductance and exchange coupling of two ferromagnets separated by a tunneling barriers", Phys. Rev, B vol. 39, pp. $6995-7002,1989$. J. C. Slonczewski, Recent Advances in Magnetism and Magnetic Materials, pp. 285-295 (World Scientific, Singapore, 1990)

[9]R. Harris, M. Plischke and. M. J. Zuckermann, "New model for amorphous magnetism", Phys. Rev. Lett., vol. 31, pp. 160-162, 1973.

[10]E. Callen, Y.J. Liu and J. R. Callen, "Initial magnetization, remanence, and coercivity of random anisotropy amorphous ferromagnet", Phys. Rev., B, vol. 16, pp. 263-268, 1977.

[11]C. B. Duke, Tunneling in Soilds (Academic, New York, 1969).

[12]T. S. Plaskett, P. P. Freitas, N. P. Barradas, M. F. da Silva and J. C. Soares, J. Appl. Phys., 76, pp. 6104-6106, 1994 